\title{
Diagnose und Management fieberhafter Harnwegsinfektionen im Säuglings- und Kleinkindesalter - AAP-Guidelines 2011
}

Im August 2011 veröffentlichte die American Academy of Pediatrics (AAP) ihre aktuelle Guideline zur Diagnostik und Therapie von Harnwegsinfektionen im Kindesalter [1]. Sie ist eine Neuauflage der im Jahr 1999 zuletzt erstellten AAP-Leitlinie, die angesichts der zwischenzeitlichen fundamentalen Kenntnisgewinne dringend überarbeitungsbedürftig erschien [2]. Bewusst beschränkte sich das Subkomitee wieder auf die Altersgruppe der 2 Monate bis 2 Jahre alten Säuglinge und Kleinkinder und auf die Erkennung, Diagnose und Therapie der ersten fieberhaften Harnwegsinfektion. Ergänzt wird die Guideline durch einen „Technical Report“ mit einer umfassenden Literaturübersicht und mit einer Metaanalyse randomisierter, kontrollierter Studien zur antibakteriellen Infektionsprophylaxe. Diese ist v.a. deshalb wertvoll, weil ihr Fokus - nach Einholen unpublizierter, altersselektiver Daten der Studien-Autoren - auf dem Altersspektrum der Leitlinien-Zielgruppe liegt [3]. Der anschließende Kommentar setzt sich kritisch mit den Leitlinien auseinander.

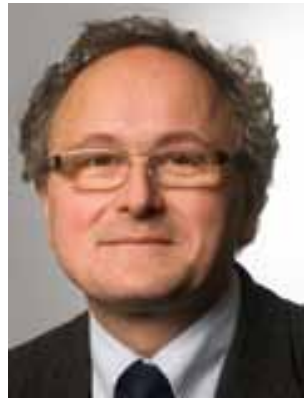

PD Dr. Rolf Beetz ist Ärztlicher Leiter der Pädiatrischen Nephrologie am Zentrum für Kinderund Jugendmedizin, Universitätsmedizin der Johannes GutenbergUniversität Mainz

Die Leitlinien-Kommission formuliert ihre Empfehlungen als Statements unter Nennung des Evidenzgrads. Unterschieden wird zudem zwischen „Empfehlung“ (recommendation) und „ausdrücklicher Empfehlung“ (strong recommendation). Sie sind im Folgenden sinngemäß aufgeführt.

\section{AAP-Guidelines 2011 \\ $\nabla$}

Statement 1

Wenn man* sich angesichts eines fiebernden Kindes ohne offensichtliche Fieberursache wegen dessen beeinträchtigten Allgemeinzustands oder anderer dringlicher Gründe zu einer antibakteriellen Therapie entscheidet, so sollte man sicherstellen, dass vor Gabe des Antibiotikums eine Urinprobe sowohl für die Urinanalyse als auch für eine Urinkultur gewonnen wird. Die Uringewinnung erfolgt mittels transurethralem Katheter oder Blasenpunk- tion. Dies ist notwendig, weil die Kulturergebnisse aus einem „Beutelurin“ keine verlässliche Diagnose einer Harnwegsinfektion erlauben.

(Evidenzgrad: A; ausdrückliche Empfehlung)

\section{Statement 2}

Falls man* ein fieberndes Kind ohne offensichtliche Fieberursache als nicht so krank einschätzt, dass es eine unmittelbare antibakterielle Therapie benötigt, sollte man die Wahrscheinlichkeit einer Harnwegsinfektion abschätzen (Beurteilungskriterien siehe 0 Tab. 1a und b).

\section{Statement 2a}

Falls man* das Vorliegen einer Harnwegsinfektion bei dem fiebernden Säugling/Kleinkind als unwahrscheinlich betrachtet, so ist eine Verlaufsbeobachtung ohne weitere Urindiagnostik ausreichend.

(Evidenzgrad: A; ausdrückliche Empfehlung).

\section{Statement 2b}

Falls man* feststellt, dass das Kind nicht der Low-Risk-Gruppe zuzuordnen ist, gibt es zwei Optionen (Evidenz: A; ausdrückliche Empfehlung):

- Option 1: Uringewinnung mittels Katheter oder Blasenpunktion für Urinanalyse und -kultur.

- Option 2: Uringewinnung mit der praktikabelsten Methode und Durch- führung einer Urinanalyse. Falls die Urin-Analyse Hinweise für eine Harnwegsinfektion gibt (positive Leukozytenesterase oder Nitritnachweis im Streifentest, Leukozyten oder Bakterien im Mikroskop), dann sollte eine Urinprobe mittels Katheterisierung oder Blasenpunktion gewonnen und eine Urinkultur durchgeführt werden

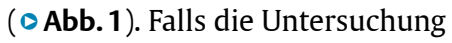
eines frischen Urins ( $<1$ Stunde seit Miktion) negative Befunde für Leukozytenesterase und Nitrit erbringt, dann ist vertretbar, den weiteren klinischen Verlauf zu beobachten, ohne dass eine antibakterielle Therapie eingeleitet wird; allerdings muss beachtet werden, dass eine negative Urinanalyse eine Harnwegsinfektion nicht sicher ausschließt.

\section{Statement 3}

Zum Nachweis einer Harnwegsinfektion sind sowohl der positive Befund in der Urinanalyse (Leukozyturie und/oder Bakteriurie), als auch eine Zahl von mindestens 50000 CFUs (colony-forming units)/ $\mathrm{mL}$ eines uropathogenen Erregers in einer durch Katheter oder Blasenpunktion gewonnenen Urinprobe erforderlich.

\section{Statement 4}

Statement 4a

Bei der Therapieeinleitung sollte man* die Applikationsform des Antibiotikums nach 
fiebernder Säugling oder Kleinkind

2 Monate - 2 Jahre,

falls keine dringliche Behandlungsindikation

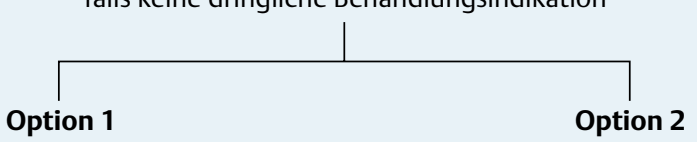

suprapubische Blasenpunktion oder Katheterurin
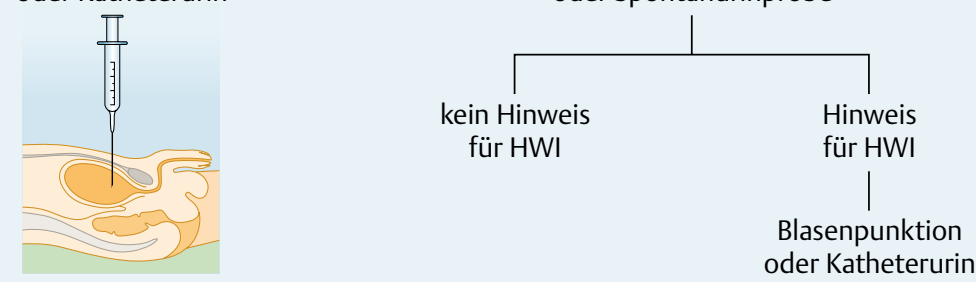

Abb.1 Vorgehen bei Verdacht auf fieberhafte Harnwegsinfektion (keine dringliche Behandlungsindikation).

praktischen Erwägungen festlegen. Parenterale und orale Therapie sind in der Effektivität gleichwertig. Die Wahl des Antibiotikums sollte die regionalen Resistenzverhältnisse (falls verfügbar) berücksichtigen; sie ist entsprechend dem Resistenzmuster des isolierten Uropathogens gegebenenfalls anzupassen.

(Evidenzgrad: A; ausdrückliche Empfehlung)

\section{Statement 4b}

Die Dauer der antibakteriellen Therapie sollte 7-14 Tage betragen.

(Evidenzgrad: B; Empfehlung)

\section{Statement 5}

Fiebernde Säuglinge mit Harnwegsinfektionen sollten eine Sonografie der Nieren und Harnblase erhalten.

(Evidenzgrad: C; Empfehlung)

\section{Statement 6}

Statement 6a

Ein Miktionszystourethrogramm sollte nicht routinemäßig nach der ersten fieberhaften Harnwegsinfektion durchgeführt werden. Ein Miktionszystourethrogramm ist dann indiziert, wenn die Ultra- schalluntersuchung eine Nierenbeckenkelchsytem-Dilatation (engl. „hydronephrosis“), narbige Parenchymveränderungen oder andere Befunde erbringt, welche einen hochgradigen vesikorenalen Reflux (VUR) oder eine obstruktive Uropathie nahelegen, sowie bei atypischen oder komplexen klinischen Verhältnissen.

(Evidenzgrad B; Empfehlung)

\section{Statement 6b}

Eine weiterführende Abklärung sollte beim Rezidiv einer fieberhaften Harnwegsinfektion erfolgen.

(Evidenzgrad X; Empfehlung)

Im Falle des Nachweises einer Harnwegsinfektion sollte man* Eltern bzw. Erziehungsberechtigte instruieren, bei zukünftigen Fieberepisoden eine rasche medizinische Abklärung (idealerweise innerhalb von 48 Stunden) anzustreben und dadurch sicherstellen, dass Rezidive erkannt und umgehend behandelt werden können. (Evidenzgrad: C; Empfehlung)

\footnotetext{
* im Originaltext erscheint hier jeweils „der Kliniker“ (the „clinician“)
}

\section{Statement 7}

\section{Kommentar}

$\nabla$

Die neuen Empfehlungen tragen den Ergebnissen von zahlreichen prospektiven, kontrollierten Studien Rechnung, die im zurückliegenden Jahrzehnt einen Kenntniszuwachs insbesondere zu den folgenden drei Aspekten erbracht haben:

- Eine orale antibakterielle Therapie ist der parenteralen antibakteriellen Therapie der Pyelonephritis hinsichtlich Keim-Eradikation und Verhinderung pyelonephritischer Nierennarben gleichwertig [4-6].

- Die Bedeutung des vesikoureteralen Refluxes für die Entstehung pyelonephritischer Parenchymschäden ist geringer als früher angenommen.

- Die antibakterielle Infektionsprophylaxe hat in mehreren Studien keine Effektivität hinsichtlich der Verhinderung von Harnwegsinfektionen und Nierenparenchymnarben gezeigt [7-10].

\section{Indikation zur Urinuntersuchung beim fiebernden Kind}

Entgegen der vorangegangenen Leitlinie sieht die jetzige Guideline nicht bei jeglichem fiebernden Kind mit ungeklärter Fieberursache eine Indikation zur Urindiagnostik. Vom betreuenden Arzt wird vielmehr eine Risikoabwägung erwartet, von welcher die Entscheidung zur Urinuntersuchung abhängig gemacht werden soll. Die Basis dieser Risikoabwägung beschränkt sich auf wenige, vorwiegend epidemiologisch begründete Kriterien ( $\odot$ Tab. 1a und b). Dieses Vorgehen verringert wahrscheinlich die Zahl falsch-positiver Urinbefunde, jedoch um den Preis einiger unentdeckt bleibender HWI.

\section{Kriterien für die Diagnose einer Harnwegsinfektion}

Eine der größten Fehlerquellen bei der Diagnostik von HWI in der angesprochenen Altersgruppe ist eine unsachgemäße Uringewinnung. Blasenpunktion oder Kathete-
Tab.1a Individuelle Risikofaktoren für HWI bei Mädchen.

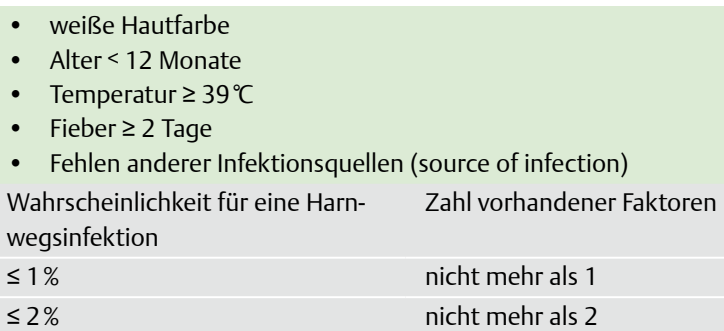

\begin{tabular}{|c|c|c|}
\hline \multicolumn{3}{|c|}{$\begin{array}{l}\text { - } \quad \text { nicht schwarzhäutig („nonblack race“) } \\
\text { - } \text { Temperatur } \geq 39^{\circ} \mathrm{C} \\
\text { - } \quad \text { Fieber }>24 \text { Stunden } \\
\text { - Fehlen anderer Infektionsquellen (source of infection) }\end{array}$} \\
\hline \multirow{2}{*}{$\begin{array}{l}\text { Wahrscheinlichkeit für eine Harn- } \\
\text { wegsinfektion }\end{array}$} & \multicolumn{2}{|c|}{ Zahl vorhandener Faktoren } \\
\hline & $\begin{array}{l}\text { nichtzir- } \\
\text { kumzidiert }\end{array}$ & zirkumzidiert \\
\hline$\leq 1 \%$ & a & nicht mehr als 2 \\
\hline$\leq 2 \%$ & keine & nicht mehr als 3 \\
\hline
\end{tabular}


risierung werden daher von den Autoren als „Goldstandard“ favorisiert. Ihnen ist jedoch bewusst, dass ein solch invasives Vorgehen kaum bei jedem Kind mit unklarem Fieber praktikabel ist. Daher wird eingeräumt, dass bei klinisch nicht dringlich erscheinender Behandlungsindikation zunächst ein „Beutelurin“ für Streifentest und Mikroskopie ausreicht - im Falle einer Leukozyturie und/oder eines mikroskopischen Bakteriennachweises ist dann jedoch eine invasive Uringewinnung für die Mikrobiologie notwendig.

Letztendlich entscheidend für die Diagnose der Harnwegsinfektion ist und bleibt der mikrobiologische Befund. Vor Einleitung der kalkulierten Therapie wird daher das Anlegen einer Urinkultur als unerlässlich angesehen. Entgegen der schon immer arbiträren „signifikanten Keimzahl“ von $\geq 100000 / \mu \mathrm{L}$ wählte man jetzt einen Grenzwert von 50000/ $\mu \mathrm{L}$, um die Sensitivität des Tests zu erhöhen und wohl auch, um der Tatsache Rechnung zu tragen, dass in dieser Altersgruppe eine Pyelonephritis angesichts kurzer Blasenverweilzeiten auch mit geringeren Keimzahlen einhergehen kann [11].

Der herabgesetzte Grenzwert ist ein Novum und wird sicher nicht unbestritten bleiben. Kürzlich machte eine angesehene britische Arbeitsgruppe den Vorschlag, die Keimzahl zugunsten einer geringeren Rate falsch-positiver Befunde sogar heraufzusetzen. Die Autoren konnten nachweisen, dass dadurch in ihrer Studienkohorte kein Fall einer Pyelonephritis unentdeckt geblieben wäre [12].

Überraschend ist der Verzicht auf jegliche Blutuntersuchung (z.B. Leukozytenzahl, Differenzialblutbild, CrP, Procalcitonin). Immerhin hat sich bei nachgewiesener Bakteriurie das Procalcitonin für die Diagnose einer Pyelonephritis als Parameter mit höchster Sensitivität und Spezifität erwiesen [13]. Stattdessen stehen klinische Einschätzung und Urinbefund ganz im Vordergrund der Diagnostik. In der restriktiven Haltung gegenüber Blutentnahmen decken sich die Empfehlungen mit den im Jahr 2007 herausgekommenen britischen NICE-Guidelines [14].

In den letzten Jahren wurde insbesondere von skandinavischen Arbeitsgruppen vorgeschlagen, durch einen DMSA (Di-mercapto-proprionyl-acid)-Scan in der Akutphase der HWI eine Nierenparenchymbeteiligung nachzuweisen, von dessen $\mathrm{Er}$ - gebnis die Indikation zur Refluxprüfung abgeleitet werden soll [15-17]. Man erhofft sich von diesem Vorgehen eine erhebliche Einsparung von Miktionszystourethrogrammen: Bei negativem DMSA-Befund empfehlen die Autoren, auf das MCU zu verzichten, weil in solchen Fällen das Vorliegen eines höhergradigen, behandlungsbedürftigen VUR extrem unwahrscheinlich ist [16]. Die AAP-Leitlinien schließen sich dieser international diskutierten Strategie nicht an. Stattdessen wird der DMSA-Scan ausdrücklich im Zusammenhang mit einer ersten fieberhaften HWI nicht empfohlen, da sein Ergebnis nur selten das akute klinische Management beeinflusst und die Strahlenbelastung zu einem möglicherweise ebenfalls notwendigen MCU addiert würde.

\section{Therapie}

Nachdem in den letzten 10 Jahren mehrere Studien eine Gleichwertigkeit der oralen antibakteriellen Therapie gegenüber der intravenösen Behandlung nachweisen konnten [4-6], überlässt die neue AAPLeitlinie die Entscheidung über die Applikationsform ganz dem behandelnden Arzt nach „praktischen Erwägungen“. Sie werden leider nicht weiter ausgeführt; man darf jedoch vermuten, dass elterliche Compliance, Notwendigkeit einer stationären Behandlung (aus welchen Gründen auch immer) und organisatorische Gegebenheiten eine Rolle spielen. Empfohlen wird ausdrücklich, bei septischem (,toxic“) Krankheitsbild und v. a. dann, wenn eine zuverlässige orale Aufnahme nicht möglich ist, eine parenterale Behandlung einzuleiten, die baldmöglichst auf eine orale Therapie umgestellt werden sollte.

In einem der Publikation vorangestellten Editorial in der gleichen Ausgabe von Pediatrics wird bedauert, dass die Leitlinienkommission nicht noch viel pointierter die orale Therapie und nur bei klinischen Kontraindikationen die i.v.-Behandlung empfohlen hat [18]. In diesem Zusammenhang werden Kostengründe aufgeführt - ein in Amerika gewichtiges Argument, das auch das Prozedere hierzulande zunehmend bestimmt. Bislang wird in Deutschland noch im Konsens empfohlen, einen kranken, fiebernden Säugling mit V.a. Pyelonephritis zumindest im ersten Lebenshalbjahr bevorzugt stationär und parenteral in einer Kinderklinik zu behandeln [19].

Angesichts der aktuellen Studienlage fasst die Leitlinienkommission ihre Empfeh- lung für die Dauer der Therapie mit 7-14 Tagen recht weit; die NICE-Guidelines legten sich demgegenüber auf 10 Tage fest [14]. Es gibt keine Unterscheidung zwischen komplizierter und unkomplizierter HWI; auch die Schwere des Krankheitsverlaufs oder das Alter des Kindes finden keine besondere Berücksichtigung - all diese Faktoren werden jedoch bei der Auslegung der AAP-Empfehlung zur individuellen Entscheidung beitragen. Sie soll offenkundig dem behandelnden Arzt überlassen werden. Dies gilt auch für die Wahl der Antibiotika, die an die jeweilige regionale Resistenzsituation uropathogener Erreger anzupassen ist.

\section{Bildgebende Diagnostik 4.1. Sonografie}

Die Ultraschalluntersuchung gehört vielerorts zur Basisdiagnostik bei erster HWI im Säuglings- und Kleinkindsalter [20, 21]. Aus Kostenerwägungen und wegen ihrer Verfügbarkeitsgrenzen ist die Sonografie als Routine-Diagnostik bei der 1 . HWI v. a. in den USA umstritten - nicht zuletzt, weil sie nur in 1-2\% der Fälle Befunde erbringen soll, die das akute therapeutische Vorgehen beeinflussen [22-26]. Auch aufgrund der zunehmenden intrauterinen Detektionsrate gravierender Harntraktanomalien wird immer wieder darüber diskutiert, bei der ersten HWI auf sie zu verzichten [27-29].

Hinsichtlich ihrer Empfehlung einer obligatorischen sonografischen Untersuchung bleiben sich die AAP-Leitlinien jedoch trotz des relativ niedrigen Evidenzniveaus treu; im Kommentar zum Statement 5 wird zudem ausgeführt, dass insbesondere bei fehlendem Ansprechen auf die antibakterielle Behandlung schnellstmöglich ein sonografischer Befund erhoben werden sollte. Für das weitere diagnostische Vorgehen erhält die Sonografie aktuell sogar eine herausragende Bedeutung, weil von ihrem Ergebnis die weiterführende bildgebende Diagnostik abhängig gemacht wird.

\subsection{Refluxdiagnostik}

Der Verzicht auf die obligatorische Refluxprüfung nach der ersten fieberhaften Harnwegsinfektion stellt einen Paradigmenwechsel dar. Während sie im Jahr 1999 unverzichtbar erschien, ist ihre Indikation nun auf diejenigen Fälle beschränkt, in welchen die Sonografie morphologische Hinweise auf einen dilatierenden VUR erbracht hat ( $\bullet$ Tab. 2 ). 
Tab. 2 Wesentliche Unterschiede zwischen früherer und aktueller AAP-Leitlinie.

\begin{tabular}{|c|c|c|}
\hline & AAP 1999 & AAP 2011 \\
\hline Fieber unklarer Genese & immer Urin-Untersuchung & $\begin{array}{l}\text { Urin-Untersuchung bei V.a. Harnwegsinfektion unter Be- } \\
\text { rücksichtigung von Risikofaktoren ( } 0 \text { Tab. 1a und b) }\end{array}$ \\
\hline $\begin{array}{l}\text { Kriterien für die Diagnose einer Harn- } \\
\text { wegsinfektion }\end{array}$ & Urinkultur & $\begin{array}{l}\text { positive Befunde in der Urinanalyse (Leukozyten und/ } \\
\text { oder Bakteriennachweis) und Keinmzahl } \geq \mid=50000 / \mu l\end{array}$ \\
\hline antibakterielle Therapie & $\begin{array}{l}\text { bei Dehydration, septischen Zeichen und Unfä- } \\
\text { higkeit zur oralen Medikamenten-Einnahme } \\
\text { parenterale Therapie; anderenfalls oral oder in- } \\
\text { travenös }\end{array}$ & $\begin{array}{l}\text { Entscheidung über orale vs. intravenöse Therapie nach } \\
\text { „praktischen klinischen Erwägungen“, ansonsten wie bei } \\
\text { AAP } 1999\end{array}$ \\
\hline $\begin{array}{l}\text { Miktionszystourethrogramm/Radion- } \\
\text { uklidzystografie }\end{array}$ & $\begin{array}{l}\text { obligatorisch. Falls kein Ansprechen auf die } \\
\text { Therapie innerhalb von } 2 \text { Tagen, Refluxprüfung } \\
\text { zum frühestmöglichen Zeitpunkt }\end{array}$ & $\begin{array}{l}\text { nicht obligatorisch. Indikationen: NBKS-Dilatation*, Par- } \\
\text { enchymdefekte, sonografische Hinweise für VUR oder } \\
\text { obstruktive Uropathien, bei „atypischen“ oder komple- } \\
\text { xen klinischen Befunden, bei fieberhaftem HWI-Rezidiv }\end{array}$ \\
\hline Sonografie & obligatorisch & $\begin{array}{l}\text { obligatorisch. Falls kein Ansprechen auf die Therapie in- } \\
\text { nerhalb von } 2 \text { Tagen, Sonografie zum frühestmöglichen } \\
\text { Zeitpunkt }\end{array}$ \\
\hline DMSA-Scan in der Akutphase & $\begin{array}{l}\text { Bedeutung für die Behandlung unklar; keine } \\
\text { Empfehlung }\end{array}$ & $\begin{array}{l}\text { nicht empfohlen in der Routinediagnostik. In Studien } \\
\text { brauchbar zum sicheren Nachweis der Pyelonephritis }\end{array}$ \\
\hline DMSA-Scan im Verlauf & $\begin{array}{l}\text { Bedeutung für die Behandlung unklar; keine } \\
\text { Empfehlung }\end{array}$ & nicht empfohlen als Teil der Routinediagnostik \\
\hline antibakterielle Infektionsprophylaxe & $\begin{array}{l}\text { nach Initialtherapie bis zum Abschluss der bild- } \\
\text { gebenden Diagnostik }\end{array}$ & nicht empfohlen \\
\hline
\end{tabular}

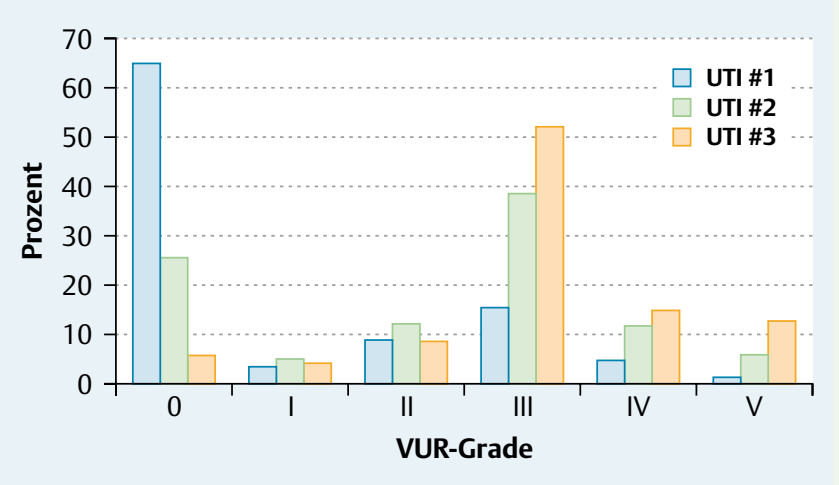

Abb. 2 Verteilung der Refluxgrade nach 1., 2. und 3. Harnwegsinfektion (nach Abb. 6 aus [3]).

Diese Restriktion lässt sich durch den zwischenzeitlich grundlegenden Wandel in der Einstellung zum vesikorenalen Reflux erklären. Man weiß heute, dass der Großteil von Parenchymdefekten, die bei höhergradigem VUR im DMSA-Scan entdeckt werden, Veränderungen im Sinne einer „konnatalen Refluxnephropathie“ entsprechen. Ein VUR führt zu keiner wesentlichen Erhöhung des HWI-Risikos selbst, sondern ist in erster Linie ein Risikofaktor für die Entstehung von persistierenden postpyelonephritischen Narben [30]. Es mögen u. a. diese Gründe gewesen sein, die zur Abkehr vom MCU als Basisdiagnostik nach erster fieberhafter HWI geführt haben. Daneben war eine kalkulatorische Überlegung dafür entscheidend, erst beim fieberhaften Rezidiv eine Refluxprüfung anzustreben: Mit der Zahl der vorangegangenen Rezidive nimmt erfahrungsgemäß die Detektionsrate dilatierender VUR erheblich zu ( $\bullet$ Abb. 2).
In einer von DGU, GPN (Gesellschaft für Pädiatrische Nephrologie) und PEG (Paul Ehrlich-Gesellschaft) gemeinsam getragenen Empfehlung wird für das Säuglingsund Kleinkindsalter eine Indikation zum MCU bereits nach erster (fieberhafter) HWI gesehen [19]. Die Argumente dafür sah die Konsensusgruppe u. a. in der weit höheren Detektionsrate höhergradiger VUR und in der erhöhten Vulnerabilität gegenüber erworbenen Parenchymschäden in dieser Altersstufe. Zudem ist im Individualfall kaum zu klären, ob früher aufgetretene, antibakteriell behandelte oder spontan rückläufige Fieberepisoden bereits unerkannten „ersten Harnwegsinfektionen" entsprechen. Hobermann et al. zeigten in einer vielbeachteten Studie, dass die Ultraschalldiagnostik einen begrenzten Vorhersagewert für einen dilatierenden VUR hat [23], sodass durcheinen „normalen“ Ultraschallbefund ein VUR nicht ausgeschlossen werden kann. Die deutsche Konsensusgruppe legt zu- dem Wert auf eine sorgfältige Familienanamese: Bei 30\% der Geschwisterkinder und $66 \%$ der Kinder von Eltern mit VUR muss mit einem VUR gerechnet werden; die Inzidenz nimmt mit zunehmendem Alter jedoch stark ab [31, 32]. Dieser Risikofaktor findet in der AAP-Leitlinie keine Berücksichtigung.

Die Aussagen der neuen AAP-Leitlinie zur Refluxdiagnostik sind aber auch auseinem anderen, rein pragmatischen Grund nicht unproblematisch: Die „erste“ Pyelonephritis im Säuglings- und Kleinkindsalter wird in Deutschland meist in einer Kinderklinik stationär behandelt. Dies bietet die Chance der Refluxdiagnostik unmittelbar nach Therapie im stationären oder nachstationären Setting. Schon diese wird bei Weitem nicht regelmäßig genutzt. In diesem Zusammenhang ist die Beobachtung einer aktuellen Studie, dass von den zur ambulanten MCU einbestellten Kindern lediglich $77 \%$ zur geplanten Untersuchung erschienen [33]. Es ist also zweifelhaft, inwieweit nach der zweiten, oft dann ambulant behandelten Pyelonephritis ein MCU tatsächlich aus der kinderärztlichen Praxis heraus veranlasst und von den Eltern wahrgenommen wird.

Im derzeitigen Praxisalltag weicht man in den USA bereits jetzt schon oft von den seit 1999 gültigen AAP-Leitlinien ab: In einer nationalen US-amerikanischen Studie zeigte sich, dass lediglich die Hälfte aller Kinder mit erster Harnwegsinfektion 


\begin{tabular}{|c|c|c|c|c|c|}
\hline $\begin{array}{l}\text { Verordnung einer antibakteriellen } \\
\text { Prophylaxe }\end{array}$ & VUR Grad I & VUR Grad II & VUR Grad III & VUR Grad IV & VUR Grad V \\
\hline nie & $30,2 \%$ & $7,1 \%$ & $2 \%$ & $2 \%$ & $3,5 \%$ \\
\hline gelegentlich, oft, oder ziemlich oft & $49,6 \%$ & $47,5 \%$ & $24,8 \%$ & $12,4 \%$ & $9,5 \%$ \\
\hline immer & $20,2 \%$ & $45,3 \%$ & $73,2 \%$ & $85,6 \%$ & $87 \%$ \\
\hline
\end{tabular}

im 1. Lebensjahr entsprechend der AAPLeitlinien behandelt wurde. Nicht hospitalisierte Kinder erhielten signifikant seltener eine bildgebende Diagnostik (relatives Risiko [RR] 1,38 für die Sonografie; 1,62 für die Refluxprüfung) [34].

Wie groß die Verunsicherung derzeit bei Pädiatern noch ist, lassen die Ergebnisse einer Umfrage bei 354 australischen Pädiatern erkennen, die ihre jeweilige persönliche Einschätzung auf einer Skala von 0-10 angeben konnten: Während sie die Indikation zum MCU bei einem 2 Monate alten Kind mit fieberhafter HW als hundertprozentig einschätzten, sahen die Pädiater eine Wahrscheinlichkeit von $55 \%$, dass ein MCU im Fall eines Dreijährigen mit gleicher Diagnose indiziert sei [35]. Aufschlussreich ist die weite Streubreite der individuellen Antworten: Es gab Einschätzungen zwischen 8 und $98 \%$ (Interquartile Range, IQR)!

\section{Antibakterielle Prophylaxe}

Bei einer kürzlich durchgeführten Umfrage zur antibakteriellen Infektionsprophylaxe unter fast 700 kanadischen Kinderärzten fanden sich große Unterschiede in der Verordnungspraxis ( 0 Tab. 3). Eindeutig war jedoch der Trend zur Prophylaxe bei höheren Refluxgraden. Nur 15\% der befragten Pädiater meinten, dass ihre Vorgehensweise evidenzbasiert sei [36]!

In den AAP-Leitlinien wird eine antibakterielle Prophylaxe nicht empfohlen; es gibt dazu in dieser Leitlinie im Unterschied zur vorangegangenen Guideline jedoch auch kein ausdrückliches Statement (๑ Tab.2). Sehr ausführlich beschäftigt sich die Kommission stattdessen in ihrem „Technical Report“ mit der antibakteriellen Infektionsprophylaxe beim VUR. So wird die bislang fehlende bzw. unsichere Evidenz für ihren Nutzen als eines der Argumente gegen die obligatorische Durchführung der Refluxprüfung angeführt (s.o.) [3].
Im Gegensatz zu den AAP-Leitlinien legt sich die American Urology Association (AUA) in ihrer Leitlinie zum Management des vesikoureteralen Refluxes eindeutig bei Säuglingen < 1 Jahr auf die Empfehlung einer antibakteriellen Infektionsprophylaxe nach 1 HWI fest [37]. Da sich die AUALeitlinie lediglich auf das Prozedere nach Diagnosestellung des VUR bezieht, gibt es keine klare Aussage zur Indikation zum MCU in dieser Altersgruppe. Die Formulierung „continuous antibiotic prophylaxis is recommended for the child with VUR with a history of a febrile UTI“ lässt jedoch durchaus den Rückschluss zu, dass aus Sicht der AUA

- a) ein MCU nach 1. fieberhafter HWI und

- b) eine antibakterielle Prophylaxe im Fall der Refluxdiagnose in diesem Alter angebracht ist.

Für ältere Kinder gilt in der AUA-Leitlinie die Empfehlung einer Prophylaxe bei Harnwegsinfektionen und VUR bei gleichzeitig bestehenden Blasenfunktionsstörungen; eine Prophylaxe wird als Option jedoch auch für solche Kinder offengehalten, die keine Blasenfunktionsstörung aufweisen [37]. Tatsächlich beurteilt die AUA die derzeitige Datenlage zur antibakteriellen Prophylaxe wesentlich zurückhaltender als die AAP und warnt vor einer überschnellen Generalisierung von Studienergebnissen, die keine Evidenz für den Nutzen der Prophylaxe zeigen konnten [37].

Derzeit erscheint die Datenlage zur antibakteriellen Infektionsprophylaxe noch zu ambivalent, als dass sich die Leitlinienkommission der AAP grundsätzlich gegen sie ausgesprochen hätte. Neuere PCT belegen immerhin eine - wenn auch relativ geringe - Effektivität bei höhergradigem VUR, und die Ergebnisse einer der größten Studien stehen derzeit noch aus [38-40]. So zählen die Autoren das ,biologisch plausible“ Konzept der Prophylaxe weiter zu den zukünftigen Forschungsaufgaben. Sie halten geeignete, gut verträgliche Harn- desinfektiva für erstrebenswert, die keine Resistenzentwicklung induzieren [3].

\section{Fazit}

In zwei entscheidenden Punkten weichen die aktuellen AAP-Leitlinien vom bisher empfohlenen Prozedere erheblich ab. Zum einen wird der Refluxdiagnostik nach der ersten fieberhaften Harnwegsinfektion kein Stellenwert mehr beigemessen. Zum anderen sehen die neuen Leitlinien keinen Benefit in einer medikamentösen antibakteriellen Infektionsprophylaxe. Gerade diese beiden Aspekte werden bis heute ausgesprochen kontrovers diskutiert. Dementsprechend haben die neuen AAPLeitlinien in den USA nicht nur Zustimmung gefunden $[41,42]$.

Die AAP-Leitlinien zur Harnwegsinfektion erschienen im August 2011, die der AUA zum Refluxmanagement im Jahr 2010. Ein größerer Konsens der beiden Gesellschaften zur Problematik der antibakteriellen Infektionsprophylaxe und zu Refluxdiagnostik wäre wünschenswert gewesen. Neue AWMF-Leitlinien zur Diagnostik, Therapie und Prophylaxe kindlicher Harnwegsinfektionen und zum vesikorenalen Reflux stehen in Deutschland aus. Der Wert der neuen AAP-Leitlinie ist dabei für die Entscheidungsfindung ohne Zweifel in vielen Punkten unschätzbar. Ohne den Konsens zwischen Kinderurologen, urologisch tätigen Kinderchirurgen, Kindernephrologen, Allgemeinpädiatern und Radiologen sind sie allerdings nicht zu machen. Die Aussichten dafür sind allerdings gut, zumal die Zahl der Studien wächst, die eine evidenzbasierte Empfehlung für die Verhältnisse im deutschsprachigen Raum ermöglichen werden.

\section{PD Dr. Rolf Beetz, Mainz}

Korrespondenz:

rolf.beetz@unimedizin-mainz.de

Literatur online unter:

www.thieme-connect.com/ejournals/toc/uro 